\title{
Failure of balloon dilatation of the pulmonary valve in carcinoid pulmonary stenosis
}

\author{
S C D Grant, J H Scarffe, R D Levy, N H Brooks
}

\footnotetext{
Wythenshawe Hospital, Manchester S C D Grant R D Levy N H Brooks CRC Department of Medical Oncology, University of Manchester J H Scarffe

Correspondence to: Dr SCD Grant, Department of Cardiology, Wythenshawe Hospital, Manchester M23 9LT

Accepted for publication 10 February 1992
}

\begin{abstract}
Background-Carcinoid heart disease typically results in pulmonary stenosis and tricuspid incompetence. Percutaneous balloon dilatation is an effective treatment for congenital pulmonary stenosis and has been applied successfully to tricuspid stenosis caused by carcinoid heart disease. The value of balloon dilatation of the pulmonary valve in carcinoid pulmonary stenosis was assessed.

Methods-Two patients with severe congestive heart failure secondary to carcinoid heart disease and with documented pulmonary stenosis had balloon dilatation of the pulmonary valve. In both cases tricuspid regurgitation was also present together with reduced cardiac output.

Results-The procedure was technically successful in both patients. One patient experienced symptomatic benefit for two months and the other experienced no improvement. Both patients subsequently required combined tricuspid and pulmonary valve replacement from which good results and symptomatic improvement were obtained.

Conclusion-Though balloon dilatation of the pulmonary valve is technically feasible it is unlikely to provide useful palliation in carcinoid heart disease. Valve surgery should be considered in patients in whom the malignancy is controlled but carcinoid heart disease is producing drug resistant congestive heart failure.
\end{abstract}

Pulmonary and tricuspid valve disease caused by the carcinoid syndrome is a rare cause of congestive heart failure. The usual pattern, of predominantly pulmonary stenosis and tricuspid incompetence, results in a considerable reduction in cardiac output. We describe two patients with carcinoid heart disease who were treated with balloon dilatation of the pulmonary valve.

\section{Case reports}

\section{CASE 1}

A 51 year old man was referred with a four month history of severe exertional dyspnoea (New York Heart Association grade III) and ankle swelling which had been resistant to diuretic therapy (frusemide $80 \mathrm{mg}$ daily and amiloride $10 \mathrm{mg}$ daily). He had presented four years earlier with diarrhoea, flushing, and palpitation and had been diagnosed as having metastatic carcinoid disease. He had undergone hepatic artery ligation and was receiving subcutaneous $\alpha$ interferon three times a week.

Examination showed a raised jugular venous pressure with a prominent $\mathrm{V}$ wave, parasternal lift, ejection murmur in the pulmonary area with a single second sound, ankle oedema, pulsatile hepatomegaly, and ascites. Echo and Doppler cardiography showed severe dilatation of the right side of the heart with tricuspid incompetence and pulmonary stenosis. The instantaneous pulmonary gradient estimated from the Doppler examination was $16 \mathrm{~mm} \mathrm{Hg}$.

Cardiac catheterisation showed a stenotic pulmonary valve with a simultaneous peak to peak right ventricular to pulmonary artery gradient of $20 \mathrm{~mm} \mathrm{Hg}$, tricuspid incompetence, and a reduced cardiac output as reflected by a pulmonary artery oxygen saturation of $60 \%$ (giving a cardiac output of $3.11 / \mathrm{min}$ calculated by the Fick formula, using assumed oxygen uptake of $117 \mathrm{ml} / \mathrm{min}^{-1} \mathrm{~m}^{-2}$ ). There was minimal tricuspid stenosis with a gradient (of $2 \mathrm{~mm} \mathrm{Hg}$ ) only during the "a" wave.

Percutaneous balloon dilatation of the pulmonary valve was performed. The valve was easily dilated with two inflations of a $19 \mathrm{~mm}$ balloon. No deformity of the balloon was seen during inflation. The gradient was abolished but right ventricular angiography showed persistent thickening and deformity in the region of the valve and there was no change in the pulmonary artery oxygen saturation, suggesting that no immediate improvement in cardiac output had occurred.

The patient experienced no benefit from the procedure and was subsequently referred for surgery without further haemodynamic assessment. At operation the pulmonary valve was found to be stenosed with fibrosed rolled back cusps and the tricuspid valve was stenosed and incompetent with fibrotic leaflets and shortened chordae adherent to the ventricular walls. Pulmonary and tricuspid valve replacement was performed without incident and with resolution of the congestive cardiac failure. The patient's treatment was subsequently changed to octreotide (a somatostatin analogue) and he was well at the time of his last assessment 18 months later.

\section{CASE 2}

A 46 year old man was referred with a six month history of severe exertional dyspnoea. He had presented three years previously with abdominal pain and had been diagnosed as suffering from carcinoid syndrome with hepatic metastases. He had been treated 
initially with $\alpha$ interferon and subsequently with octreotide.

On examination he had a raised jugular venous pressure with a prominent $V$ wave, soft pulmonary component of the second heart sound, and ejection murmur in the pulmonary area with a quiet early diastolic murmur at the left sternal edge. Abdominal examination showed tender hepatomegaly.

Echocardiography showed tricuspid incompetence and pulmonary stenosis with incompetence and mild aortic incompetence. Cardiac catheterisation showed a simultaneous peak to peak gradient of $40 \mathrm{~mm} \mathrm{Hg}$ across the right ventricular outflow tract. There was mild tricuspid stenosis (reflected by an "a" wave gradient of $5 \mathrm{~mm} \mathrm{Hg}$ ), gross tricuspid incompetence on right ventricular angiography, and a cardiac output of $3.2 \mathrm{l} / \mathrm{min}$, calculated by the Fick formula with an assumed oxygen consumption of $118 \mathrm{ml} / \mathrm{min}^{-1} \mathrm{~m}^{-2}$ ).

Percutaneous dilatation of the pulmonary valve was undertaken with a $20 \mathrm{~mm}$ balloon followed by a $23 \mathrm{~mm}$ balloon. As in case 1 no nipping of the balloon was observed during inflation. The gradient across the valve was reduced to $15 \mathrm{~mm} \mathrm{Hg}$ though the angiogram showed persistent thickening and there was no improvement in the pulmonary artery oxygen saturation. The procedure produced immediate symptomatic improvement and the clinical signs of tricuspid regurgitation disappeared.

Unfortunately the improvement was maintained for only two months, when he relapsed. Repeat catheterisation showed recurrence of the pulmonary gradient with a withdrawal gradient of $38 \mathrm{~mm} \mathrm{Hg}$ and right ventricular pressure of $55 / 5 \mathrm{~mm} \mathrm{Hg}$. In view of these findings and the clinical deterioration he was referred for surgery. At operation the pulmonary valve cusps were heavily thickened and rolled up against the valve ring. The tricuspid valve was grossly disorganised with the posterior cusp heavily thickened and rolled backwards. The tricuspid and pulmonary valves were replaced and he was comparatively well 14 months later.

\section{Discussion}

\section{CARCINOID SYNDROME}

The carcinoid syndrome is produced by the metabolic products, as yet incompletely identified, of a slowly progressive malignancy usually originating in the small bowel (although occasionally from other sites such as ovary or lung). The syndrome is produced only when the tumour has metastasised to liver, presumably because before this the metabolites are produced in small quantities and are detoxified by the liver. Lesions of the heart valves are reported to occur in about $20 \%$ of cases clinically, ${ }^{1}$ but if echocardiography is used they are detected in up to $70 \% .^{23}$ There is conflicting evidence about the overall importance of cardiac disease as a cause of death in the carcinoid syndrome: Moertel stated that only $10 \%$ of patients with the carcinoid syndrome developed cardiac symptoms and of these half died a cardiac death (about $5 \%$ of patients with the syndrome), ${ }^{1}$ Thorson et al reported that $50 \%$ of patients have cardiac failure causing death in $30 \%$ of patients, ${ }^{4}$ Lundin et al that "right ventricular failure is one of the major causes of death in patients with the carcinoid syndrome resulting from midgut tumours," and Ross and Roberts that "carcinoid heart disease frequently is the cause of death in patients with the carcinoid syndrome". ${ }^{6}$ The explanation for this divergence of opinion is not clear, though there may have been differences in the patient populations and, as discussed below, with improved control of the malignancy heart failure may have become an increasingly common problem.

The disease typically affects the right side of the heart: plaques are occasionally found in the left heart but they are rarely of haemodynamic significance.

\section{PATHOLOGY}

The anatomical consequences of involvement of the tricuspid and pulmonary valves are narrowing of the valve orifices together with tethering and immobility of the leaflets. Possibly because the two valves are of different sizes this produces principally incompetence with a much lesser degree of stenosis in the tricuspid valve and principally stenosis with a minor degree of incompetence in the pulmonary valve. ${ }^{67}$ The combination of outflow tract obstruction with atrioventricular valvar incompetence is liable to have a profound effect on cardiac output with the pulmonary stenosis exacerbating the tricuspid regurgitation.

Pathological studies have looked at the distribution and nature of the plaques and there has been speculation about the pathogenesis. ${ }^{6-9}$ In a necropsy study of 21 cases the plaques were found to affect the valve structures and also the endocardial surfaces of the right atrium and the right ventricle, though right ventricular plaques were smaller (both in thickness and area). Plaques were also sometimes seen in the great veins and coronary sinus. ${ }^{6}$ Histology showed that the atrial and ventricular surfaces of the anterior leaflet of the tricuspid valve were involved in the fibrotic process, but only the ventricular surface of the posterior and septal leaflets of the tricuspid valve and the pulmonary arterial surface of the pulmonary valve.

Ultrastructural studies of the carcinoid plaques have proved interesting. ${ }^{810}$ It was initially thought that the plaques were of collagen and fibrin but it has been shown by light and electron microscopical studies that they are composed of smooth muscle cells (thought to be derived from primitive mesenchymal cells present normally in the subendocardial layer) in a stroma of acid mucopolysaccharide, collagen, and microfibrils. No elastic fibres are present. ${ }^{810}$ The lesions are often predominantly superficial and well circumscribed and they were described in the earlier work ${ }^{8}$ as being entirely limited to the endocardium and subendocardial layer. However, the more recent study ${ }^{10}$ suggested a more infiltrative nature and showed lesions extending into the myocardium. 
AETIOLOGY OF PLAQUES

The aetiology of the plaques is unknown. The clinical features of the carcinoid syndrome (flushing, diarrhoea, and bronchospasm), are thought to be produced by the effects of the circulating vasoactive products of the malignancy, including serotonin and its metabolites, tachykinins (neuropeptide $\mathrm{K}$ and substance $\mathrm{P}$ ), prostaglandins, kallikrein and bradykinin. ${ }^{811}$ There are, however, probably numerous other unidentified compounds at work. It has been postulated that the plaques are a result of the action of these substances on the endocardium and that a resulting increase in endothelial permeability allows migration of primitive mesenchymal cells which then differentiate into the smooth muscle cells seen in the carcinoid lesions. It has been further speculated that this process of endothelial injury is exacerbated by hydrodynamic factors such as turbulence, which might explain the localisation of plaques. $^{8}$

Several studies have attempted to correlate factors such as length of symptoms and concentrations of circulating substances with the presence of heart disease in the carcinoid syndrome. Most have found that the presence of carcinoid heart disease is not associated with a longer duration of symptoms ${ }^{612}$ but that it is associated with raised concentrations of circulating vasoactive compounds (serotoninreflected in the urinary concentrations of its metabolite 5-hydroxyindole acetic acid ${ }^{112}$ and tachykinins ${ }^{11}$ ), but there have been conflicting findings. ${ }^{1314}$ Though raised concentrations have not been shown to be associated with carcinoid heart disease, bradykinin has also been proposed as an aetiological factor, because it is produced in large amounts, is capable of producing endothelial injury, and its inactivation in the lung might explain the predominance of lesions in the right side of the heart. $^{89}$

Evidence also suggests a link between serotonin rich diets and endomyocardial fibrosis in Uganda. ${ }^{15}$ Finally methysergide (a serotonin antagonist) can produce retroperitoneal and occasionally valvar and endocardial lesions, ${ }^{16}$ both of which point indirectly to the role of serotonin in the aetiology of the plaques.

PROGNOSIS AND THE NEED FOR INTERVENTION By the time carcinoid heart disease has developed the prognosis is poor with a median survival time of 14 months. In the past most patients died of their malignancy or other complications rather than heart disease. ${ }^{1}$ In general, therefore, carcinoid heart disease has been treated conservatively, surgery being reserved for patients thought likely to have an unusually good prognosis. Recently introduced medical treatments have greatly improved the quality of life for many with the carcinoid syndrome. Both $\alpha$ interferon and octreotide (a long acting somatostatin analogue) reduce flushing and diarrhoea in $60-70 \%$ of patients, and there is some evidence of improved survival with $\alpha$ interferon. ${ }^{17} 18$ If patients survive longer with a better quality of life the relative impact of the cardiac lesions is likely to be greater and it can be expected that the number of patients in good general condition referred for cardiological intervention will increase in the next few years.

We argued that although the pulmonary valve gradients were not great in our two patients, relief of the outflow tract obstruction would reduce the degree of tricuspid regurgitation and improve cardiac output. The dramatic resolution of the tricuspid incompetence in case 2-albeit for only a few weeks-provides some support for this proposition.

Balloon dilatation of the pulmonary valve is a safe technique, with excellent immediate and long-term results in patients with congenital pulmonary valve stenosis. It is the recommended treatment for patients with this condition. ${ }^{19}$

Successful balloon dilatation of carcinoid tricuspid stenosis has been reported in one patient and the authors speculated that balloon dilatation of carcinoid pulmonary stenosis was also likely to be effective. ${ }^{20}$ The likely efficacy of balloon dilatation applied to the carcinoid pulmonary valve has also been speculated upon by Cheng. ${ }^{21}$ Combined balloon dilatation of the tricuspid and pulmonary valves has also been reported as successful in a patient with combined tricuspid and pulmonary stenosis in the carcinoid heart, ${ }^{22}$ not the typical pattern of valve lesions in this disease.

The results in our two patients, however, were disappointing; whereas double valve replacement produced good results. The rapid recurrence of the right ventricular outflow tract obstruction is perhaps not surprising in view of the pathology. Presumably the balloon merely compressed the fibrous plaques, which subsequently recoiled or continued to grow, in contrast to the splitting of fused commissures that is caused by balloon dilatation for pulmonary valve stenosis. The smaller pulmonary valve gradient in case 1 may indicate that in this case the tricuspid regurgitation was a more important cause of congestive cardiac failure than in case 2 .

Reported cases of valve surgery for carcinoid heart disease include a series of 13 patients collected from published reports and reviewed by Strickman ${ }^{9}$ and a series of 32 reported cases collected more recently by Lundin et al. ${ }^{5}$ Further cases have also recently been described by Bilinska et $a^{23}$ and by Fetherston and Davis. ${ }^{24}$ Patients undergoing surgery (usually tricuspid valve replacement with either pulmonary valvotomy, valvectomy or valve replacement ${ }^{5}$ ) have in general done well, with the median survival from Strickman's series being in excess of two years, which is excellent given the natural cause of the disease by the time cardiac symptoms develop.

The two cases described here accord with the view that valve surgery can be expected to produce good symptomatic relief in advanced carcinoid heart disease and is superior to balloon dilatation of the pulmonary valve, which we found to be ineffective.

We thank Mr AN Rahman who performed the surgery in both cases. 
1 Moertel CG. Treatment of the carcinoid tumour and the malignant carcinoid syndrome. J Clin Oncol 1983;11: $727-40$.

2 Lundin L, Norheim I, Landelius J, Öberg K, TheodorssonNorheim E. Carcinoid heart disease: relationship of
circulating vasoactive substances to ultrasound detectable cardiac abnormalities. Circulation 1988;77:264-9.

3 Lundin L, Landelius J, Andrén B, Öberg K. Transoesophageal echocardiography improves the diagnostic value phageal echocardiography improves the diagnostic value
of cardiac ultrasound in patients with carcinoid heart of cardiac ultrasound in patients
disease. $B r$ Heart $J 1990 ; 64: 190-4$.

4 Thorson A, Biorck G, Bjorkman G, Waldenstrom J. Malignant carcinoid of the small intestine with metastasis to the liver, valvular disease of the heart (pulmonary stenosis and tricuspid regurgitation without septal defects) peripheral vasomotor symptoms, bronchoconstriction and pathologic syndrome. Am Heart J 1954;47: 796-817.

5 Lundin L, Hansson HE, Landelius, Öberg K. Surgical treatment of carcinoid heart disease. $J$ Thorac Cardiovasc Surg 1990;100:552-61.

6 Ross EM, Roberts WC. The carcinoid syndrome: comparison of 21 necropsy subjects with carcinoid heart disease to 15 necropsy subiects without carcinoid heart disease. $A m$ Med 1985;79:339-53.

7 Trell E, Rausing A, Ripa J, Porp A, Waldenstrom J. Carcinoid heart disease; Clinicopathological findings and Carcinoid heart disease; Clinicopathological finding

8 Ferrans VJ, Roberts WC. The carcinoid endocardial plaque, an ultrastructural study. Hum Pathol 1976;7:387-409.

9 Strickman NE, Rossi PA, Massum Kharis GA, et al. Carcinoid heart disease. Current Prob Cardiol 1982;6:1-41.

10 Lundin L, Funa K, Hansson HE, Wilander E, Öberg K. Histochemical and immunohistochemical morphology of carcinoid heart disease. Pathol Res Pract 1991;187:73-7.

11 Lundin L, Norheim I, Landelius J, Oberg K, TheodorssonNorheim E. Carcinoid heart disease: relationship of circulating vasoactive substances to ultrasound-detecta cardiac abnormalities. Circulation 1988;77:264-9.
12 Himelman RB, Schiller NB. Clinical and echocardiographic comparison of patients with the carcinoid syndrome with and without carcinoid heart disease. Am J Cardio 1989;63:347-52.

13 Arora RR, Warner RRP. Do indole markers predict carcinoid heart disease. Chest 1986;90:87-9.

14 Roberts WC, Sjoerdsma A. The cardiac disease associated with the carcinoid syndrome (carcinoid heart disease). $A m$ J Med 1964;36:5-34.

15 McKinney B, Crawford MA. Fibrosis in guinea pig diet produced by plantain diet. Lancet 1965;ii:880-5.

16 Graham JR. Methysergide for prevention of headache Experience in five hundred patients over three years. $N$ Experience in five hundred

17 Öberg K, Norheim I, Lind E, et al. Treatment of malignant carcinoid tumours with human leukocyte interferon. Long-term results. Cancer Treat Rep 1986;70:1297-304.

$18 \mathrm{Kvols}$ LK, Moertel CG, O'Connoll MS, et al. Treatment of malignant carcinoid syndrome, evaluation of a long acting somatostatin analogue. $N$ Engl J Med 1986;315:663-6.

19 Fontes VF, Sousa JEMR, Esteves CA, et al. Pulmonary valvoplasty-experience of 100 cases. Int J Cardiol 1988; 21:335-42.

20 Mullins PA, Hall JA, Shapiro LM. Balloon dilatation of tricuspid stenosis caused by carcinoid heart disease. $\mathrm{Br}$ Heart J 1990;63:249-50.

21 Cheng TO. Nonsurgical treatment of carcinoid heart disease. Ann Thorac Surg 1991;51:1046-7.

22 Alcibar VJ, Gochi IR, Onate LA, Inguanzo BR, Alvarez BA Barreiro GG. Tricuspid and pulmonary percutaneous valvuloplasty in the cardiopathy of the carcinoid synvalvuloplasty in the cardiopathy of the carcinoid syn-
drome. A case report. Rev Esp Cardiol 1991;44:238-7.

23 Bilinska ZT, Miskiewicz Z, Rozanski J, et al. Carcinoid heart disease. One-year survival following tricuspid valve replacement and pulmonary valvotomy. CVWorld Report 1990;3:173-8.

24 Fetherston GJ, Davis BB. Surgical management of carcinoid heart disease. Ann Thorac Surg 1991;51:493-4. 